\title{
Mycobacterial infection in patients infected with the human immunodeficiency virus
}

\author{
M Helbert, D Robinson, D Buchanan, T Hellyer, M McCarthy, I Brown, A J Pinching, \\ D M Mitchell
}

the UK AIDS epidemic (1983) until March 1988. Chest radiographs were reviewed blind and independently by two radiologists in conjunction with chest radiographs from patients with other HIV related conditions. Data on BCG vaccination were insufficient for analysis.

Data were obtained from two sources: (1) the records of 207 patients with AIDS attending St Mary's Hospital; (2) a study of the prevalence of carriage of stool mycobacteria in 63 homosexual and bisexual men (39 with asymptomatic HIV infection and 24 with the AIDS related complex).

Smears from all specimens were stained for acid fast bacilli with auramine. Kirschner medium was inoculated (solid samples were not crushed) and incubated for up to 14 weeks. Growth of mycobacteria was confirmed in cultures that became turbid with auramine staining. If positive, these were subcultured on Lowenstein-Jensen medium. Blood samples for culture of mycobacteria were taken into Dupont isolators. Lysed blood samples, stool specimens, and bronchoalveolar lavage fluid were treated as above as well as being cultured by the Bactec radiometric method. ${ }^{10}$ Necropsy material was not available.

Indications for treatment in individual cases are outlined below. The likely benefits were discussed before treatment was initiated. Despite the frequency of side effects and of other medication, compliance appeared to be good. Patients attended for follow up at least monthly.

After review of the case notes nontuberculous mycobacterial isolates that did not appear to be related to clinical disease were considered to be contaminants.

\section{Results}

\section{Mycobacterium tuberculosis}

Tuberculosis was established bacteriologically in 15 homosexual and bisexual patients without a history of intravenous drug abuse. All had symptoms; fever (11 patients) and respiratory symptoms (10 patients) were common. Six patients had diarrhoea, four lymphadenopathy, and two hepatomegaly. Extrapulmonary tuberculosis in an HIV seropositive individual is now a diagnostic criterion for AIDS ${ }^{11}$ and was the first reason to diagnose AIDS in four patients (table 1). Two patients with the AIDS related complex presented with pulmonary tuberculosis limited to the lungs; one progressed to AIDS within five months, when he developed cryptosporidiosis; and the other is

\section{Methods}

Clinical, bacteriological, and treatment data were collected from case notes from the start of 
Table 1 Site of Mycobacterium tuberculosis infection in 207 patients with AIDS and 24 with AIDS related complex

\begin{tabular}{llll}
\hline & $\begin{array}{l}\text { Gastro- } \\
\text { intestinal }\end{array}$ & Lung & $\begin{array}{l}\text { Dissem- } \\
\text { inated }\end{array}$ \\
\hline AIDS related complex & & 2 & \\
First AIDS infection & 2 & 4 & 2 \\
Previous AIDS diagnosis & 2 & 4 & 3 \\
\hline
\end{tabular}

clinically stable. For the nine patients who had previously had a major opportunist infection, such as Pneumocystis carinii pneumonia, or Kaposi's sarcoma, the mean time between the diagnosis of AIDS and the development of tuberculosis was 5.4 (range 2-12) months.

Seven chest radiographs from patients with pulmonary or disseminated disease without copathogens were available for review; two were normal and three showed cavities, two reticulonodular shadowing, and five air space shadowing. Reticulonodular shadowing or cavitation in the upper zone was considered suggestive of $M$ tuberculosis infection. On the basis of these criteria, four of the seven chest radiographs suggested tuberculosis.

Positive cultures were obtained from bronchoalveolar lavage fluid (5), transbronchial biopsy material (3), lavage fluid and transbronchial biopsy material (2), stool (6), lymph node biopsy material (2), pleural fluid, lavage fluid, stool, and marrow aspirate (1). Acid fast bacilli were never seen on direct examination of bronchoalveolar lavage fluid. Coinfections with $P$ carinii, Staphylococcus, and Haemophilus influenzae each occurred in one patient; all were diagnosed from bronchoalveolar lavage fluid.

All $M$ tuberculosis isolates tested were sensitive to streptomycin and isoniazid and all except one to rifampicin and ethambutaol. Eleven patients were treated with rifampicin, ethambutol, isoniazid, and pyrazinamide and nine responded clinically. The two nonresponders had AIDS. Death was a direct result of tuberculosis in only one patient, who declined treatment. The median survival of the eight patients with AIDS from the time of diagnosis of tuberculosis was five months.

\section{Mycobacterium avium-intracellulare}

Isolation $M$ avium-intercellulare was isolated from 20 patients. Isolation in one patient occurred only during a bacterial chest infection, which resolved with amoxycillin alone. This isolate was considered to be a contaminant or asymptomatically carried organism.

Enteritis Seven patients had $M$ aviumintracellulare isolated from stool alone. Two had no symptoms and, of the remainder with diarrhoea or abdominal pain, two had other enteric pathogens (cytomegalovirus in one and cytomegalovirus with cryptosporidiosis in the other). Hence symptomatic enteritis apparently due to $M$ avium-intracellulare alone occurred in three patients only, all of whom had AIDS (mean duration nine months). It did not contribute directly to death in the two who died. Two patients were treated, one with rifampicin, isoniazid, ethembutol, and pyrazinamide and the other with ansamycin instead of rifampicin; neither responded clinically or microbiologically.
Table 2 Antibiotic sensitivities of Mycobacterium avium-intracellulare isolates

\begin{tabular}{lcc}
\hline & \multicolumn{2}{c}{ No of isolates } \\
\cline { 2 - 3 } Antibiotic & sensitive & tested \\
\hline Amikacin & 2 & 5 \\
Ansamycin & 7 & 9 \\
Capreomycin & 2 & 15 \\
Ciprofloxacin & 0 & 20 \\
Clofazamine & 0 & 20 \\
Cycloserine & 11 & 13 \\
Ethambutol & 0 & 20 \\
Isoniazid & 0 & 20 \\
Rifampicin & 1 & 12 \\
Streptomycin & 0 & 20 \\
\hline
\end{tabular}

Disseminated infection Isolation from two or more sites or from blood was interpreted as disseminated infection. This occurred in 12 patients with AIDS (mean time after first opportunist infection 10.4 months). All were febrile, six had diarrhoea, five cough or dyspnoea, three lymphadenopathy, and three hepatomegaly. No other pathogens were isolated to account for these features. Culture positive samples came from sputum (4) cases, liver biopsy specimens (4), lymph node biopsy specimens or aspirates (4), blood culture (3), bronchoalveolar lavage and fluid and urine (1). Acid fast bacilli were present on a direct smear of one sputum sample only (and in bronchoalveolar lavage fluid from the same patient). Four patients had pure isolates from sputum and lavage fluid. The chest radiographs showed a perihilar haze in three of these cases, which was interpreted as $P$ carinii pneumonia. One chest radiograph showed reticulonodular shadowing and cavities, attributed to mycobacterial infection. Median survival from diagnosis of disseminated $M$ avium-intracellulare infection was five months in the patients not treated.

Treatment The in vitro antibiotic sensitivities of $M$ avium-intracellulare isolates are given in table 2. Treatment was attempted in eight patients; four patients received conventional quadruple chemotherapy (rifampicin, isoniazid, ethambutol, and pyrazinamide) with no response. Two were treated with ansamycin, isoniazid, and pyrazinamide and improved clinically. The addition of amikacin produced clinical improvement in two further patients.

\section{Mycobacterium ulcerans}

$M$ ulcerans was isolated from an indolent leg ulcer in a patient with the AIDS related complex; antibiotic sensitivities were not determined. The patient was treated initially with conventional antimycobacterial drugs, but improved only when amikacin and ansamycin were given.

Table 3 Mycobacterial stool contaminants

\begin{tabular}{|c|c|c|}
\hline & $\begin{array}{l}\text { No of } \\
\text { patients } \\
\text { tested }\end{array}$ & Mycobacteria isolated \\
\hline $\begin{array}{l}\text { Asymptomatic HIV } \\
\text { infection } \\
\text { AIDS related complex }\end{array}$ & $\begin{array}{l}39 \\
24\end{array}$ & \multirow{2}{*}{$\begin{array}{l}1 \text { not typed } \\
1 M \text { avium-intracellulare } \\
1 \text { not typed } \\
1 M \text { fortuitum } \\
3 \mathrm{M} \text { avium-intracellulare } \\
3 \text { not typed }\end{array}$} \\
\hline AIDS & 87 & \\
\hline
\end{tabular}


Table 4 Isolates of mycobacteria from patients with AIDS considered to be contaminants

\begin{tabular}{ll}
\hline Source $(n)$ & Other isolates and disease \\
\hline & \multicolumn{1}{c}{ M xenopi } \\
BAL (2) & Staphylococcus aureus \\
BAL (1) & Pneumocystis carinii \\
Sputum (1) & S aureus and $P$ carinii \\
EMU (1) & Lymphoma \\
& M chelonei or $M$ fortuitum \\
BAL (2) & $P$ carinii, other bacteria \\
TBB (2) & $P$ carinii, other bacteria \\
Stool (1) & Asymptomatic \\
& $M$ flavescens \\
EMU (2) &
\end{tabular}

BAL_bronchoalveolar lavage fluid; EMU-early morning urine; TBB-transbronchial biopsy specimen

\section{Mycobacterium kansasii}

$M$ kansasii was isolated from retroperitoneal lymph node material and from pleural fluid from a patient with AIDS and fever, dyspnoea, and weight loss. He recovered partially with rifampicin, ethambutol, isoniazid, and pyrazinamide, but died of progressive HIV encephalopathy.

Ten stool isolates were considered contaminants (table 3). This included the four $M$ avium-intracellulare isolates discussed above. Three further isolates were cultured from stools of patients without gastrointestinal symptoms (one with asymptomatic HIV infection and two with AIDS). In three patients with gastrointestinal symptoms other pathogens were isolated. One patient with the AIDS related complex had salmonellosis and two patients with AIDS had cytomegalovirus colitis. Symptoms improved in all cases without treatment directed at the mycobacterium isolated. These mycobacterial isolates were considered to be contaminants.

Twelve other isolates were cultured from various other sites, usually as part of a screen investigating pyrexia of unknown origin. They were also regarded as contaminants because other pathogens were found to account for the symptoms (table 4 ). None was smear positive at the initial examination.

\section{Discussion}

Tuberculosis occurred in $6 \%$ of the patients with AIDS. The prevalence of HIV infection in British patients with tuberculosis is unknown but in the United States up to $12 \%$ of patients with tuberculosis have HIV infection..$^{12}$ Intravenous drug abuse was a risk factor for tuberculosis before the human immunodeficiency virus epidemic. ${ }^{13}$ Patients with a history of drug abuse account for a small but increasing number of cases of AIDS at St Mary's Hospital, so the prevalence of tuberculosis in our patients may increase. Atypical pulmonary or disseminated tuberculosis suggests the possibility of underlying HIV infection. Owing to lack of data on prior BCG inoculation it is not clear whether tuberculosis in these patients was due to reactivation or reinfection.

Tuberculin testing of patients with HIV disease is unhelpful as many are anergic. ${ }^{14}$ Radiological features of pulmonary tuberculosis in our patients were frequently atypical or absent. This small survey suggests that bronchoalveolar lavage may be useful for confirming the diagnosis of tuberculosis and excluding copathogens.

The patients with tuberculosis who were treated conventionally with four agents usually showed a good clinical response; the two patients who did not respond well both had AIDS, suggesting that a satisfactory response was limited by profound immunosuppression. Sunderam ${ }^{15}$ reported a patient with tuberculosis treated with rifampicin, isoniazid, and pyrazinamide who, after a good clinical response, had an extrapulmonary relapse after pyrazinamide had been stopped. The patient was subsequently found to have HIV infection. Treatment with three or even four agents would seem to be most appropriate in this setting, and lifelong treatment with isoniazid has been recommended. ${ }^{16}$ Similarly, it may be judicious to offer chemoprophylaxis to HIV positive individuals with a history of tuberculosis or recent exposure.

Treatment difficulties are compounded by side effects, which are more common in HIV infected patients, and by concurrent multiple opportunist infections or neoplasms requiring complex treatment regimens. ${ }^{17}$ For example, deterioration in visual acuity may be due to ethambutol retinopathy or cytomegalovirus retinitis.

$M$ tuberculosis infection might hasten disease progression in HIV infected individuals by activating $\mathrm{CD} 4$ positive ("helper") lymphocytes and increasing virus replication. Patients with tuberculosis who are HIV antibody positive should perhaps be considered early for treatment with zidovudine.

We identified homosexual men infected with HIV who were symptomless stool carriers of mycobacteria. The suggestion that sexual practices might explain the tendency for $M$ avium-intracellulare to cause primarily gastrointestinal disease in homosexual patients with AIDS seems unlikely as stool carriage of this organism occurs in healthy heterosexuals, ${ }^{19}$ though the prevalence in this group is unknown. An alternative explanation is that $M$ avium-intracellulare infection in patients not infected with HIV frequently affects old tuberculous cavities, so that this group by contrast has a propensity for pulmonary disease. A positive stool culture in an HIV positive patient may imply little more than the need to search for $M$ avium-intracellulare from sources other than the lung in symptomatic patients.

Changes on the chest radiographs of patients with $M$ avium-intracellulare infection were not specific and tended to suggest $P$ carinii pneumonia. Confirmation of $M$ avium-intracellulare infection was obtained more frequently from sputum than by bronchoscopy. Obtaining a blood culture positive for $M$ aviumintracellulare was a convenient non-invasive method of proving disseminated infection. A system that lyses white blood cells is required as most organisms are intracellular. Radiometric systems such as Bactec provides positive results within 14 days.

In an American series patients with AIDS 
who were infected with $M$ avium-intracellulare did not have a worse prognosis than those with no such infection; the mean survival was $8 \cdot 2$ months for patients with pneumocystis pneumonia and 10.2 months for those infected with $M$ avium-intracellulare in addition to $P$ carinii (P Demopulos et al, paper presented to the 25th Interscience Conference on Antimicrobial Agents and Chemotherapy, Minneapolis, 1985). This was not so in our study, though numbers of patients were small; the mean survival after diagnosis of $M$ aviumintracellulare infection was five months compared with 12 months after diagnosis of pneumocystis pneumonia. This may reflect greater immunosuppression in patients with $M$ avium-intracellulare. Infection was associated with symptoms and contributed to death. Attempts at treatment are therefore justified.

$M$ avium-intracellulare isolates from patients with AIDS show increased antimicrobial resistance. ${ }^{20}$ Ansamycin was useful in four cases and amikacin in two cases. These agents may also be effective in treating the intercurrent Gram negative bacteraemia that may be present in these patients ${ }^{2122}$ and may have clinical effects due to actions other than their antimycobacterial effects. Treatment with ansamycin reduces the yield of $M$ avium-intracellulare in blood culture, ${ }^{23}$ and the drug is also active in vitro against $\mathrm{HIV},{ }^{24}$ which might explain the clinical findings. Ethambutol in vitro acts synergistically with both rifampicin and ansamycin on some strains of $M$ avium-intracellulare. ${ }^{25}$

In our series $M$ tuberculosis and $M$ aviumintracellulare acounted for most mycobacterial infection in patients with AIDS and other HIV related diseases. Other mycobacterial isolates were usually considered to be contaminants as other pathogens were found to account for symptoms.

1 Snider DE, Hopewell PE, Mills J, Reichman LB. Mycobacterioses and the acquired immunodeficiency syndrome. Am Rev Respir Dis 1987;136:492-6.

2 Goedert JJ, Weiss SH, Biggar AJ, et al. Lesser AIDS and tuberculosis. Lancet 1985; ii:52.

3 Schecter $G$, Rutherford $G$, Echenberg $D$. Tuberculosis in AIDS patients in San Francisco, 1982-1985 [abstract] Am Rev Respir Dis 1986;133(suppl):A184.
4 Sunderam G, McDonald RJ, Maniatis T, et al. Tuberculosis as a manifestation of acquired immunodeficiency syndrome (AIDS). JAMA 1986;256:362-6.

5 Meeran K. Prevalence of HIV infection among patients with leprosy and tuberculosis in rural Zambia. Br Med J 1989;298:364-5.

6 National Heart, Lung and Blood Institute. Pulmonary complications of the Acquired Immunodeficiency complications of the Acquired Immunodeficiency Syndrome-report of a national heart, lung and
institute workshop. $N$ Eng J Med 1984;310:1682-8.

7 Armstrong D, Gold JWM, Dryjanski JM, et al. Treatmen of infections in patients with the Acquired Immunodeficiency Syndrome. Ann Intern Med 1985;103:738-43.

8 Shaw RJ, Rowland-Hill CA, Parkin J, et al. Pulmonary complications of AIDS: a review of 90 English cases [abstract]. Thorax 1986;41:730.

9 Millar AB. Respiratory manifestations of AIDS. Br J Hosp Med 1988;39:204-13.

10 Macher AM, Kovacs JA, Gill V, et al. Bacteraemia due to mycobacterium avium intracellulare in AIDS. Ann Intern Med 1983;99:782-5.

11 Centers for disease control. Revision of the CDC surveillance case definition for acquired immunodeficiency syndrome. Morbidity and Mortality Weekly Report 1987;36(suppl 1S):3-13.

12 Chaisson RE, Schetcter EF, Theuer CP, et al. Tuberculosis in patients with the acquired immunodeficiency syndrome. Am Rev Respir Dis 1987;136:570-4.

13 Reichman LB, Felton CP, Edsall JR. Drug dependence, possible new risk factor for tuberculosis disease. Arch Intern Med 1979;139:337-9.

14 Pinching AJ, McManus TJ, Jeffries DJ, et al. Studies of cellular immunity in male homosexuals in London. Lancet 1983;ii:126-30.

15 Sunderam G, Mangura BT, Lombardo JM, et al. Failure of "optimal" four drug short course tuberculosis chemotherapy in a compliant patient with human immunodeficiency virus. Am Rev Respir Dis 1987136:1475-8. 16 Iseman MD. Is standard chemotherapy adequate in
tuberculosis patients infected with HIV? Am Rev Respir Dis 1987;136:1326.

17 Englehard D, Stutman HR, Marks MI. Interaction of ketoconazole with rifampicin and isoniazid. $N$ Engl J Med 1984:309:945-50.

18 Damsker B, Bottone EJ. Mycobacterium avium intracellulare from the intestinal tracts of patients with the acquired immunodeficiency syndrome: concepts regarding acquisiimmunodeficiency syndrome: concepts regarding acqu

19 Graham DY, Markesich DC, Yoshima HH. Mycobacteria and inflammatory bowel disease. Gastroenterology 1987;92:436-42.

20 Horsburgh CR, Cohn DL, Roberts RL, et al. Mycobacterium Avium-M intracellulare isolates from patients with or without the acquired immunodeficiency syndrome. Antimicrob Agents Chemother 1986;30:955-7.

21 Pasternak J, Bolivar R, Hopfer RL, et al. Bacteraemia caused campylobacter like organism in two male homosexuals. Ann Intern Med 1984;101339-41.

22 Smith PD, Machert AM, Bookman MA, et al. Salmonell typhimurium enteritis in the acquired immunodeficiency syndrome. Ann Intern Med 1985;102:207-9.

23 Wong B, Fitzroy FE, Kiehn TE, et al. Continuous high grade mycobacterium avium intracellular bacteraemia in patients with the acquired immunodeficiency syndrome. Am J Med 1985;78:35-40.

24 Anadnd R, Moore JL, Curran JW, Srinivasan A. Interaction between rifabutin and the human immunodeficiency virus type I: inhibition of replication cytopathic effect, and reverse transcription in vitro. Antimicrob Agents Chemother 1988;32:684-8.

25 Heifets LB, Iseman MD, Lindholm-Levy PJ. Combination of rifampin or rifabutine plus ethambutol against of rifampin or rifabutine plus ethambutol against mycobacterium 